CASE REPORT

\title{
Sialometaplasia arising in the ectopic salivary gland ductal inclusions of multiple intraparotid lymph nodes
}

\author{
S Sarioğlu, U Pabuççuoğlu, C Ecevit, K Ceryan, S Paksoy, E Ada
}

J Clin Pathol 2004;57:1335-1337. doi: 10.1136/icp.2004.017202

Sialometaplasia, squamous metaplasia of salivary gland lobules, is a benign condition occasionally presenting with lesions clinically simulating malignancy. "Necrotising sialometaplasia", recognised by lobular infarction, necrosis, and simultaneous squamous metaplasia of ducts and acini is a well known condition. There are only a few reports of the "proliferative type of sialometaplasia", which is recognised by a more mature morphology of larger and more irregular metaplastic nests, lacking necrosis. This report describes a unique case of "proliferative sialometaplasia of multiple intraparotid lymph nodes" occurring in a 55 year old woman, presenting with multiple parotid lumps. This interesting case points to the importance of intraparotid lymph nodes as sites for multiple lesions of the parotid region.

$\mathrm{S}$ ialometaplasia, squamous metaplasia (SM) of salivary gland lobules, is a benign condition occasionally presenting with lesions mimicking malignancy. Since the description of the necrotising type, ${ }^{1}$ many cases have been published. The proliferative form has only been reported in a few cases in intraparotid lymph nodes (LNs). ${ }^{2}$ This type of sialometaplasia is recognised by solid squamous metaplastic nests involving ductal structures and lacking atypia and necrosis. ${ }^{2}$

\section{CASE REPORT}

A 55 year old woman presented with left infra-auricular multiple lumps, of two years duration. She had undergone stapedectomy of the right ear five years previously.

The patient had a $1.5 \times 1.5 \mathrm{~cm}$, non-tender, semimobile mass in the left preauricular region, and a similar mass in the tail of the left parotid gland. The functions of the facial nerve were bilaterally normal and the examination of the other head and neck sites including the other salivary glands was unremarkable. She had no complaints of xerostomia. Sonography revealed hypoechoic parotid nodules. The lesions were hyperdense with contrast enhancement on computed tomography scans. Magnetic resonance imaging demonstrated two spherical discrete masses. Lesions were hypointense on the $\mathrm{Tl}$ images and isointense on the $\mathrm{T} 2$ images with contrast enhancement (fig 1). The radiological conclusion was consistent with a benign tumour, in particularly pleomorphic adenoma, and the clinical diagnosis was that of pleomorphic adenoma and associated lympadenomegaly of intraparotid LNs. The routine laboratory examinations were not remarkable: white blood cell count, $8.1 \times 10^{9}$ /litre; haemoglobin, $135 \mathrm{~g} /$ litre; haematocrit, 39.3\%; erythrocyte sedimentation rate, $21 \mathrm{~mm} /$ hour; antinuclear antibodies, antineutrophil cytoplasmic antibodies, human immunodeficiency virus were negative.

Left superficial parotidectomy with preservation of the facial nerve was performed.

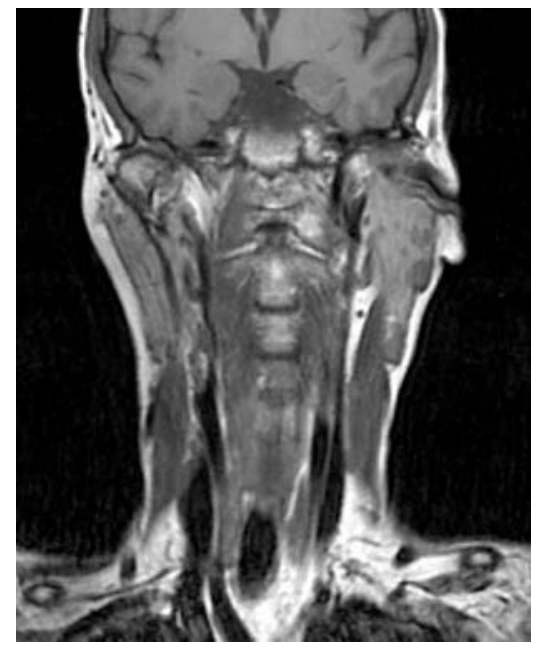

Figure 1 Coronal $\mathrm{Tl}$ weighted image; hypointense circumscribed mass lesions in the left parotid gland.

\section{Pathological examination}

Superficial parotidectomy revealed two encapsulated, $1.5 \mathrm{~cm}$, grey/white nodules located at the poles. Histology showed that they were both LNs and six additional LNs were also identified. Five LNs revealed ductal salivary gland inclusions, almost totally replaced by SM, but retaining the lobular architecture of ductal inclusions (figs 2, 3). Ductal inclusions were also identified in another lymph node. The parotid gland showed atrophic changes.

The metaplastic squamous epithelium was keratin positive and mucicarmen negative. Smooth muscle actin and S-100 was positive at the periphery of the metaplastic lobules (fig 4). The diagnosis was that of "proliferative sialometaplasia (PS)" involving salivary gland inclusions of multiple intraparotid LNs.

The patient is well 12 months after surgery.

\section{DISCUSSION}

Lymphoid and epithelial lesions may arise from intraparotid LNs, which originate from the inclusions of salivary ducts and acini. $^{2-7}$ The frequency of these lesions increases dramatically when cystadenoma lymphomatosum cases, which are thought to arise from intranodal ductal inclusions, are considered. ${ }^{8}$

Multiple lumps at this region are frequently related to lesions originating from LNs, and the leading lesion is again cystadenoma lymphomatosum. ${ }^{9}$ In our present case, histopathological examination revealed extensive SM of the

Abbreviations: LN, lymph node; PS, proliferative sialometaplasia; SM, squamous metaplasia 


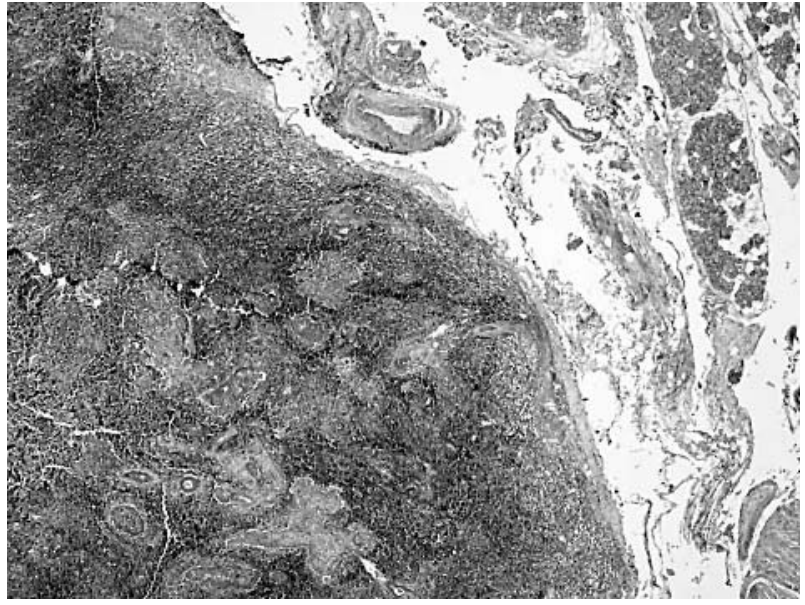

Figure 2 Extensive squamous metaplasia of the intraparotid ductal inclusions; note the capsule of the lymph node and preserved architecture of the adjacent parotid gland (haematoxylin and eosin stain; original magnification, $\times 4$ ).

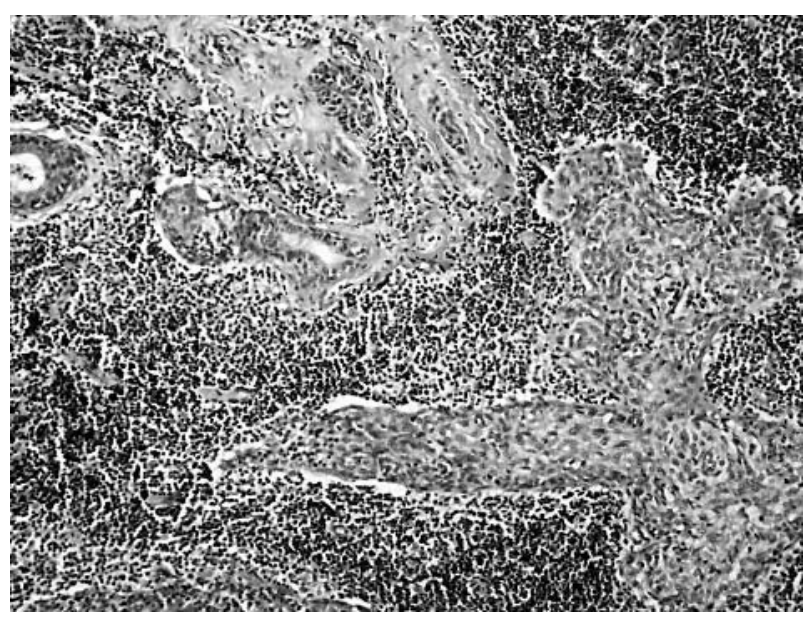

Figure 3 High power view of squamous metaplasia and nodal inclusions (haematoxylin and eosin stain; original magnification, $\times 20$ ).

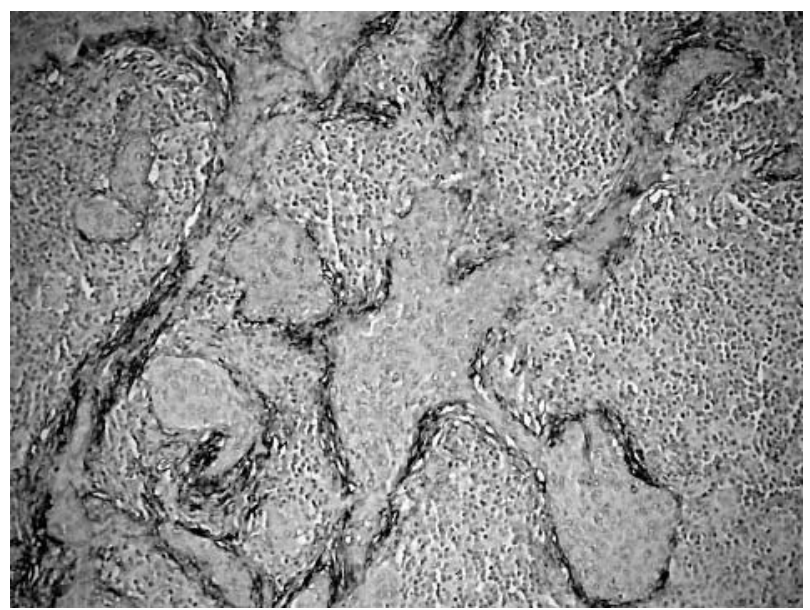

Figure 4 Smooth muscle actin expression at the periphery of the metaplastic lobules (haematoxylin and eosin stain; original magnification, $\times 10$ ).

\section{Take home messages}

- We report a unique case of proliferative sialometaplasia of multiple intraparotid lymph nodes occurring in a 55 year old woman, presenting with multiple parotid lumps

- This interesting case points to the importance of intraparotid lymph nodes as sites for multiple lesions of the parotid region

intranodal inclusions, with a preserved lobular architecture involving five intraparotid LNs. Among the previous reports we noticed cases comparable to this one. Goldman and Klein ${ }^{2}$ described PS of an intraparotid lymph node. Ryan et al described two patients with AIDS who presented with lumps at the parotid region, with lymphadenitis and SM of the salivary gland ductal inclusions. ${ }^{3}$ As in our present case, those cases did not fulfill the established criteria for the diagnosis of necrotising sialometaplasia ${ }^{1}$ described by Abrams et al, ${ }^{1}$ because we could not consistently demonstrate infarction. Anneroth and Hansen ${ }^{10}$ suggested five stages in the course of necrotising sialometaplasia, namely: infarction, sequestration, ulceration, reparation, and healing. The lesions in our patient might represent the reparation and healing stages of necrotising sialometaplasia, but without evidence of necrosis the case falls into the category of proliferative SM.

"We feel that this unique case is important because, to the best of our knowledge, this is the first case of proliferative sialometaplasia involving multiple intraparotid lymph nodes"

The differential diagnosis of proliferative SM also includes metastatic squamous cell carcinoma and mucoepidermoid carcinoma. ${ }^{21011}$ In our present case, no cellular atypia or mitotic activity was encountered, thus excluding carcinoma. The coexpression of cytokeratin, S-100, and actin was consistent with a lesion related to myoepithelial cells. The lack of chondromyxoid stroma and the presence of multiple intranodal lesions aided in the exclusion of squamous metaplasia prominent pleomorphic adenoma. ${ }^{2}$ The patient's clinical features were inconsistent with the established criteria $^{12}$ of Sjögren's syndrome and the involvement of intraparotid lymph nodes has not been described for this disease.

We feel that this unique case is important because, to the best of our knowledge, this is the first case of PS involving multiple intraparotid LNs. PS should be included in the differential diagnosis of multiple lumps of the parotid region.

\section{Authors' affiliations \\ S Sarioğlu, U Pabuççuoğlu, S Paksoy, Department of Pathology, Faculty of Medicine, Dokuz Eylül University, 35340 Izmir/Turkey \\ C Ecevit, K Ceryan, Department of Oto-Rhino-Laryngology, Head and Neck Surgery, Faculty of Medicine, Dokuz Eylül University \\ E Ada, Department of Radiodiagnostics, Faculty of Medicine, Dokuz Eylül University \\ Correspondence to: Dr S Sarıoğlu, Department of Pathology, Dokuz Eylül University School of Medicine, 35340 İnciraltı/Izmir, Turkey; sulen.sarioglu@deu.edu.tr}

Accepted for publication 22 June 2004 


\section{REFERENCES}

1 Abrams AM, Melrose RJ, Howel FV. Necrotizing sialometaplasia. A disease simulating malignancy. Cancer 1973;32:13-15.

2 Goldman RL, Klein Z. Proliferative sialometaplasia arising in intraparotid lymph node. Am J Clin Pathol 1986:86:118-19.

3 Ryan JR, lochim HL, Marmer J, et al. Acquired immunodeficiency syndrome related lymphadenopathies presenting in the salivary gland lymph nodes. Arch Otolaryngol 1985;111:554-6.

4 Pisani P, Ramponi A, Francesco P. The deep parotid lymph nodes: an anatomical and oncological study. J Laryngol Otol 1996;1 10:148-50.

5 Lopez JI, Ballestin C. Fine-needle aspiration cytology of a membranous basal cell adenoma arising in an intraparotid lymph node. Diagn Cytopathol 1993;9:668-72.

6 Adkins GF, Hinckley DM. Primary muco-epidermoid carcinoma arising in a parotid lymph node. Aust N Z J Surg 1989;5:433-5.
7 Perzin KH, Livolsi VA. Acinic cell carcinoma arising in ectopic salivary gland tissue. Cancer 1980;45:967-72.

8 Low WK, Ng SB. Synchronous parotid and nasopharyngeal Warthin's tumors: first report of a case. Ear Nose Throat J 2002;81:839-41.

9 Franzen A, Koegel K. Synchronous double tumors of the parotid gland. Laryngorhinootologie 1996;75:437-40.

10 Anneroth G, Hansen LS. Necrotizing sialometaplasia: the relationship of its pathogenesis to its clinical characteristics. Int J Oral Surg 1982;11:283-91

11 Pabuççuoğlu U, Özkara E, Sarıoğlu S. Necrotizing sialometaplasia. Report of five cases including uncommon localizations. Turkish Journal of Medical Sciences 2002;32:355-9.

12 Vitali C, Bombardieri S, Jonsson R, et al. Classification criteria for Siögren's syndrome: a revised version of the European criteria proposed by the American-European consensus group. Ann Rheum Dis 2002;61:554-8.

\section{$\mathrm{ECHO}$}

Familial frontotemporal dementia: early manifestations

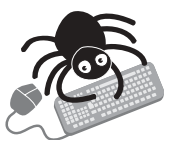

Please visit the Journal of Clinical Pathology website [www. jclinpath.com] for a link to the full text of this article. rontotemporal dementia (FTD) is second only to Alzheimer's disease as a cause of - dementia. It is mainly sporadic but may be familial, often with a mutation (P301L) in the tau gene. Behavioural and language disturbances are common and it may be associated with parkinsonism in some cases and motor neurone disease in others. The detailed findings in an asymptomatic woman with this mutation have been described from Italy.

Two of the subject's three brothers had developed FTD. Her one sister and the third brother were asymptomatic and neurologically and psychometrically normal at age 58 and 52 respectively and did not carry the mutation. The P301L mutation was demonstrated in the asymptomatic subject and her two affected brothers. At the age of 50 she was holding down a demanding job, leading an appropriate social life, and showed no behavioural abnormalities. She performed normally on a battery of cognitive tests with the single exception of the Verbal Fluency Test for letters in which she produced only 17 words in one minute compared with the 30 and 43 words of her two unaffected siblings. Brain CT and gross inspection of brain single photon emission computed tomography (SPECT) showed no abnormality but on SPECT with statistical parametric mapping (SPECT-SPM) there was reduced blood flow in the frontal lobes, particularly the dorsolateral frontal cortex and frontal poles and the mesial frontal cortex. SPECT-SM on the normal sister was within normal limits. The subject's cerebrospinal fluid (CSF) contained increased levels of A $\beta 1-42$, tau protein, and 181P-tau. (Patients with Alzheimer's disease have high tau and low A $\beta 1-42$ levels in CSF.)

Testing for the abnormalities found in this asymptomatic subject might improve early diagnostic accuracy in FTD and help to distinguish it from Alzheimer's disease.

A Journal of Neurology, Neurosurgery, and Psychiatry 2004;75:1607-1610. 